\title{
Equações Preditivas de Gordura Corporal: Saber Escolher é Fundamental
}

\section{"Carta ao Editor" referente ao artigo:}

"Consumo Alimentar e Perfil Antropométrico de Tenistas Amadores e Profissionais (Vol. 15, No 6 - Nov/Dez, 2009)"

Roberto Fernandes da Costa,

Mestre em Educação Física - USP, Doutor em Ciências Aplicadas à Pediatria - Unifesp, Diretor Científico do Centro de Estudos e Pesquisas Sanny - CEPS

O artigo intitulado: Consumo Alimentar e Perfil Antropométrico de Tenistas Amadores e Profissionais (Vol. 15, No 6 - Nov/Dez, 2009) aborda um tema de grande relevância para profissionais e estudiosos do tênis, sobretudo pela escassez de artigos científicos, produzidos em nosso país, que tratam de aspectos nutricionais e da composição corporal de tenistas. Nesse estudo, os autores avaliaram a porcentagem de gordura de dois grupos de atletas de tênis do sexo masculino, sendo um de amadores e outro de profissionais, utilizando quatro equações preditivas de densidade corporal diferentes.

Há diversas equações preditivas de densidade e gordura corporal a partir da medida de espessura de dobras cutâneas, sendo muitas delas derivadas de estudos com atletas de diferentes modalidades esportivas esportivas ${ }^{(1)}$. Assim, a escolha da equação preditiva deve levar em consideração aspectos como a etnia, o sexo, a idade, o nível de treinamento e a quantidade de gordura corporal do avaliado, a fim de aumentar a validade de predição da técnica ${ }^{(2)}$.

No estudo em questão, as equações utilizadas foram produzidas com base em estudos de sujeitos com características diferentes às de atletas de tênis, o que pode ter comprometido, significativamente, os resultados encontrados.

A equação de Jackson et al. ${ }^{(3)}$ foi criada para avaliar mulheres não atletas, o que pode explicar os menores resultados de porcentagem de gordura encontrados para os dois grupos de tenistas estudados, quando da utilização desta equação.

Durnin e Womersley ${ }^{(4)}$ produziram suas equações para a população em geral, de 16 a 74 anos de idade, do Reino Unido, ou seja, também não específicas para atletas.

Quanto às equações de Guedes(5) e Petroski(6), o primeiro autor contou, em sua amostra, com estudantes universitários do Rio Grande do Sul, e o segundo, com população de Santa Catarina e do Rio Grande do Sul, sendo que, em ambos os estudos, as equações não foram produzidas para avaliar atletas.

A utilização de equações não específicas para atletas, neste caso para tenistas, causa dúvidas quanto aos resultados encontrados no referido estudo, e pode sugerir valores de referência inadequados para atletas desta modalidade.

Sinning et al. ${ }^{(7)}$ propuseram valores de referência para atletas de diferentes modalidades esportivas, utilizando equações específicas. Para tenistas do sexo masculino os autores encontraram valor médio de porcentagem de gordura de 11,3 \pm 5,2; inferior ao encontrado nas três equações para sexo masculino do artigo em questão.

Em conclusão, sugere-se que para a avaliação da composição corporal de atletas, a escolha das equações preditivas seja feita de forma cuidadosa, considerando-se as características dos avaliados, para que seja obtida maior validade de estimativa da porcentagem de gordura corporal.

\section{REFERÊNCIAS}

1. Costa RF, Böhme MTS. Avaliação morfológica no esporte. In: Biezek S, Alves LA, Guerra I. Estratégias de nutrição e suplementação no esporte. Barueri: Manole, 2005

2. Costa RF. Composição corporal: avaliação prárica. In: Hirschbruch MD, Carvalho JR. Nutrição esportiva: uma visão prárica. $2^{\mathrm{a}}$ ed. Barueri: Manole, 2008.

3. Jackson AS, Pollock ML, Ward A. Generalized equations for predicting body density of women. Med Sci Sports Exerc 1980;12:175-82.

4. Durnin JV, Womersley J. Body fat assessed from body density and its estimation from skinfold thickness: measurements on 481 men and women aged from 16 to 72 years. Br J Nutr 1974;32:77-97.

5. Guedes DP. Estudo da gordura corporal através da mensuração dos valores de densidade corporal e espessura de dobras cutâneas em universitários. [Dissertaçăo de Mestrado]. Santa Maria (RS), Universidade Federal de Santa Maria, 1985

6. Petroski EL. Desenvolvimento e validação de equaçōes generalizadas para a estimativa da densidade corporal em adultos. [Tese de Doutorado]. Santa Maria (RS), Universidade Federal de Santa Maria, 1995. 7. Sinning WE, Dolny DG, Little KD, Cunningham LN, Racaniello A, Siconolfi SF, Sholes JL. Validity of "generalized" equations for body composition analysis in male athletes. Med Sci Sports Exerc 1985;17:124-30.

\section{Resposta}

Rodrigo Vitasovic Gomes', Sandra Maria Lima Ribeiro², Renata Furlan Veibigi3, Marcelo Saldanha Aoki²

1. Escola de Educação Física e Esporte da Universidade de São Paulo, São Paulo, Brasil.

2. Escola de Artes, Ciências e Humanidades da Universidade de São Paulo, São Paulo, Brasil.

3. Centro Universitário São Camilo, São Paulo, Brasil.

Inicialmente, agradecemos o interesse pelo nosso manuscrito (Consumo Alimentar e Perfil Antropométrico de Tenistas Amadores e Profissionais Vol. 15, No 6 - Nov/Dez, 2009), demonstrado pelo autor da Carta ao editor, intitulada "Equações Preditivas de Gordura Corporal: saber escoIher é fundamental". Agradecemos também a oportunidade para esclarecer alguns pontos relativos ao manuscrito e, adicionalmente, alguns tópicos abordados na Carta ao editor.

O comentário sobre a equação de Jackson e Pollock(1) (1980) foi, extremamente, pertinente e útil, pois nos possibilitou detectar um equívoco de nossa parte, que precisa ser esclarecido. No corpo do texto e nas referências, citamos a equação de 1980, desenvolvida para mulheres. Porém, nós utilizamos, na verdade, a equação de Jackson e Pollock(2), publicada em 1978 [1.112 - $0.00043499 \times(\Sigma 7$ dobras $)+0.00000055 \times$ $(\Sigma 7 \text { dobras })^{2}-0.00028826 \times$ (idade)]. Essa equação foi desenvolvida para estimar o conteúdo de gordura corporal de homens ${ }^{(2)}$. Portanto, houve um engano, provavelmente, em decorrência do uso do software que foi empregado para organizar a seção das referências bibliográficas. Aproveitamos essa réplica para nos desculpar e agradecer a oportunidade de esclarecer este engano.

Outro ponto destacado na carta ao editor, que deve ser considerado, diz respeito às diferentes equações preditivas para determinar o conteúdo de gordura corporal disponíveis na literatura. Concordamos que a escolha é fundamental, bem como, acreditamos que os critérios para tal escolha devem ser, apropriadamente, considerados. Neste sentido, diante do nosso desconhecimento de uma equação validada para tenistas (profissionais e amadores) brasileiros, nós decidimos utilizar algumas equações preditivas, amplamente, utilizadas em diferentes estudos. A apresentação de dados provenientes de diversas equações, além de demonstrar nossa preocupação com essa limitação, também revela as diferenças emergentes do uso destas equações preditivas. 
A despeito destas limitações, as equações preditivas de gordura corporal, adotadas no nosso manuscrito, têm apresentado validade e confiabilidade bastante razoáveis. Por exemplo, vale mencionar que a estimativa obtida através da equação de Jackson e Pollock ${ }^{(2)}$ (1978) $(8,9 \pm 3,6 \%)$, no estudo de validação publicado por Sinning et al. ${ }^{(3)}$, utilizando 265 atletas universitários, apresentou forte correlação $(r=0,902)$ com o valor aferido através da pesagem hidrostática $(9,2 \pm 4,4 \%)$.

No referido estudo, o percentual de gordura para a amostra de tenistas $(n=9)$ determinado através da pesagem hidrostática foi de $11,3 \pm 5,2^{(3)}$ Equivocadamente, foi levantado na Carta ao editor que esse percentual teria sido estimado por equações específicas. [Trecho da carta: "Sinning et al. ${ }^{(7)}$, propuseram valores de referência para atletas de diferentes modalidades esportivas, utilizando equações específicas. Para tenistas do sexo masculino os autores encontraram valor médio de porcentagem de gordura de 11,3 \pm 5,2; inferior ao encontrado nas três equações para sexo masculino do artigo em questão."]. É importante mencionar que nesse estudo(3) não foram propostos valores de referência, pois isso seria inviável com uma amostra composta por somente 9 tenistas universitários. Na verdade, o referido estudo teve como objetivo validar equações já disponíveis, a partir do método de pesagem hidrostática ${ }^{(3)}$. Logo, o valor referente aos tenistas (11,3 $\left.\pm 5,2 \%\right)$ foi aferido através da pesagem hidrostática [ver tabela 5 do estudo de Sinning et al. $\left.{ }^{(3)}\right]$ e, não pelo uso de equações específicas, como levantado na Carta ao editor. Houve, portanto, um erro de tradução e interpretação de texto por parte do autor da referida carta.

Além disto, no mesmo estudo de validação ${ }^{3}$, a equação para atletas proposta por Forsyth e Sinning ${ }^{(4)}$, através de regressão linear, superestimou o percentual de gordura em 2,4 até 3,8\% em relação ao método de pesagem hidrostática. Portanto, ao contrário do que é sugerido na "Carta ao editor", o fato da equação ter sido desenvolvida para atletas não resultou em estimativa mais próxima ao valor observado pela pesagem hidrostática.

No estudo de validação de Sinning et al.(3), das 21 equações estudadas, apenas três equações não apresentaram diferença em relação à pesagem hidrostática. Uma delas foi a equação de Jackson e Pollock ${ }^{(2)}$ (1978) que estimou valor semelhante ao observado pela pesagem hidrostática para os tenistas avaliados (Pesagem hidrostática: 11,3 \pm 5,2 vs. Jackson e Pollock (1978): 9,8 $\pm 3,4)^{(3)}$. No nosso estudo, essa mesma equação(2) estimou o percentual de gordura dos tenistas profissionais em 9,0 \pm 4,6\% e dos tenistas amadores em 9,8 $\pm 2,2 \%$. O valor observado por Sinning et al. ${ }^{(3)}$, utilizando a equação de Jackson e Pollock ${ }^{(2)}$ (1978), para os 9 tenistas norte-americamos foi de 9,8\% de gordura corporal, enquanto que, o valor médio dos 24 tenistas brasileiros, estimado pela mesma equação, no nosso estudo foi de 9,4\%. Indubitavelmente, esses dados reforçam que a escolha da equação de Jackson e Pollock ${ }^{(2)}$ (1978) é, plenamente, justificável.

Com relação à equação de Durnin e Womersley ${ }^{(5)}$, no nosso estudo foram estimados valores referentes à gordura corporal de 14,2 $\pm 4,2 \%$ para profissionais e 15,5 $\pm 1,4 \%$ para amadores. Recentemente, em um estudo conduzido por Juzwiak et al.(6), utilizando tenistas brasileiros, com faixa etária similar (14 a 18 anos) a da nossa amostra (18,0 \pm 1,4 anos), foi reportado valor médio de 14,6 \pm 7,5\% de gordura corporal aferido através do DEXA (Dual energy X-ray absorptiometry) (método padrão-ouro). Os nossos dados indicam que os valores obtidos a partir da equação de Durnin e Womersley ${ }^{(5)}$ estão muito próximos dos valores determinados pelo DEXA(6), corroborando, mais uma vez, os critérios adotados para a escolha das equações.

No tocante, às equações de Guedes ${ }^{(7)}$ e Petroski ${ }^{(8)}$, estas foram utilizadas, fundamentalmente, por terem sido desenvolvidas no Brasil. Além disto, a equação de Guedes foi desenvolvida a partir de uma população com faixa etária semelhante a dos tenistas que participaram da nossa investigação. No nosso estudo, os valores de gordura corporal estimados pela equação de Guedes $^{(7)}$ foram de 13,8 \pm 5,5\% para profissionais e 13,7 $\pm 2,4 \%$ para amadores, enquanto que os valores estimados pela equação de Petroski ${ }^{(8)}$ foram 12,3 $\pm 4,2 \%$ para profissionais e 12,8 $\pm 1,6 \%$ para amadores. Ambas as equações desenvolvidas por estes pesquisadores brasileiros, realmente, apresentaram valores "superiores" ao valor dos tenistas avaliados por Sinning et al. ${ }^{(3)}$ através de pesagem hidrostática (11,3 $\left.\pm 5,2 \%\right)$. No entanto, a análise dos resultados nos permite afirmar que esses valores são muito próximos, 0 que contrasta com a forma que o comentário-crítica foi escrito, sugerindo ao leitor que a diferença seria substancial e significativa.

Conforme já mencionamos anteriormente, no estudo de Sinning et al.(3), uma equação desenvolvida para atletas (Forsyth e Sinning ${ }^{(4)}$ ) superestimou a gor dura corporal quando comparada à pesagem hidrostática em 2,4 até 3,8\%. Esses valores percentuais (2,4 até 3,8\%) são maiores que a variação observada para as equações de Guedes $^{7}$ e Pestroski ${ }^{8}$ em comparação ao valor de 11,3 \pm 5,2\% obtido por pesagem hidrostática no estudo de Sinning et al. ${ }^{(3)}$. Logo, apesar das equações de Guedes ${ }^{(7)} \mathrm{e}$ Pestroski ${ }^{(8)}$ não terem sido desenvolvidas, especificamente, para atletas, as mesmas revelaram estimativas mais próximas do que a equação de Forsyth e Sinning ${ }^{(4)}$ avaliada no estudo de validação ${ }^{(3)}$.

Em um trecho da carta é sugerido que os resultados causam dúvida. ["A utilização de equações não específicas para atletas, neste caso para tenistas, causa dúvidas quanto aos resultados encontrados no referido estudo, e pode sugerir valores de referência inadequados para atletas desta modalidade."] Discordamos, fortemente, desse comentário. Na nossa opinião, não há dúvidas sobre os resultados do nosso estudo! Nós utilizamos diferentes equações para comparar o percentual de gordura de duas populações de atletas, e apesar das limitações de cada equação, o erro intrínseco de cada estimativa foi repetido, sistematicamente, para os dois grupos estudados. A partir desses dados foram realizadas as comparações entre os atletas amadores e os atletas profissionais. Não há dúvida nenhuma sobre este resultado.

É óbvio que o estabelecimento de valores de referência demandam um número bem maior de sujeitos. Nesse sentido, também gostaríamos de ressaltar que não tivemos a intenção de propor valores de referência para tenistas. O objetivo do manuscrito, claramente explicitado na introdução, foi comparar parâmetros de dois grupos de atletas (amadores vs. profissionais). Mais uma vez, a crítica levantada teve origem a partir de outro equívoco de interpretação, desta vez sobre a hipótese e o objetivo do nosso estudo!

Concordamos que a escolha da equação preditiva é um passo fundamental, no entanto, não temos uma equação específica para tenistas brasileiros. Por isso, resolvemos utilizar 4 equações diferentes [duas avaliadas no estudo de Sinning et al.(3)], essa nossa preocupação já deixa claro que tínhamos plena noção das limitações de cada equação. Portanto, a idéia de utilizar diferentes equações preditivas do conteúdo de gordura corporal foi fornecer um amplo panorama de comparação entre as duas populações de atletas.

Por fim, acreditamos que os pesquisadores da área e os centros de pesquisa deveriam investir tempo e concentrar esforços para desenvolver equações específicas para o contexto do esporte. Este tipo de iniciativa, no sentido de promover produção de conhecimento, ao invés de levantar polêmicas desnecessárias e apresentar críticas baseadas na interpretação imprecisa de dados, seria de grande valia para o avanço científico da área.

\section{REFERÊNCIAS}

1. Jackson AS, Pollock ML, Ward A. Generalized equations for predicting body density of women. Med Sci Sports Exerc 1980;12:175-82.

2. Jackson AS, Pollock ML, Ward A. Generalized equations for predicting body density of men. Br J Nutr 1978;40:497-504.

3. Sinning WE, Dolny DG, Little KD, Cunningham LN, Racaniello A, Siconolfi SF, Sholes JL. Validity of "generalized" equations for body composition analysis in male athletes. Med Sci Sports Exerc 1985:17:124 30.

4. Forsyth HL, Sinning WE. The anthropometric estimation of body density and lean body weight of male athletes. Med Sci Sports 1973;5:174-80. 5urnin JV, Womersley J. Body fat assessed from body density and its estimation from skinfold thickness: measurements on 481 men and women aged from 16 to 72 years. Br J Nutr. 1974;32:77-97.

6. Juzwiak CA, Amâncio OS, Vitalle MSS, Pinheiro MM, Szejnfeld VL. Body composition and nutritional profile of male adolescent tennis players. J Sports Sci 2008:26:1209-17.

7. Guedes DP. Estudo da gordura corporal através da mensuração dos valores de densidade corporal e e espessura de dobras cutâneas em universitários. [Dissertação de Mestrado]. Santa Maria (RS), Universidade Federal de Santa Maria, 1985.

8. Petroski EL. Desenvolvimento e validação de equações generalizadas para a estimativa da densidade corporal em adultos. [Tese de Doutorado]. Santa Maria (RS), Universidade Federal de Santa Maria, 1995. 